\title{
openheart Impaired autonomic function after incomplete revascularisation
}

\author{
Richard Armstrong (D) , ${ }^{1}$ Peter Wheen (D) ,2 Lisa Brandon, ${ }^{2}$ Ciarán Finucane, ${ }^{3}$ \\ Rose Anne Kenny, ${ }^{3}$ Andrew Maree ${ }^{2}$
}

To cite: Armstrong R, Wheen $\mathrm{P}$, Brandon L, et al. Impaired autonomic function after incomplete revascularisation. Open Heart 2021;8:e001835. doi:10.1136/ openhrt-2021-001835

Received 25 August 2021 Accepted 27 September 2021

Check for updates

(C) Author(s) (or their employer(s)) 2021. Re-use permitted under CC BY-NC. No commercial re-use. See rights and permissions. Published by BMJ.

${ }^{1}$ Cardiology, Belfast Health and Social Care Trust, Belfast, UK ${ }^{2}$ Department of Cardiology, St James Hospital, Dublin, Ireland ${ }^{3}$ TILDA Medical Gerontology, Trinity College, Dublin, Ireland

Correspondence to Dr Richard Armstrong; richarm88@gmail.com

\section{ABSTRACT}

Introduction Incomplete cardiac revascularisation (ICR) assessed by residual SYNTAX score (rSs) is associated with increased 5-year mortality. Furthermore, in the general population, our group has demonstrated that impaired autonomic function determined by heart rate recovery time between 10 and $20 \mathrm{~s}\left(\mathrm{HRR}_{10-20}\right)$ following an active stand is associated with increased all-cause mortality.

Purpose We hypothesised that ICR would be associated with impaired autonomic function determined by $\mathrm{HRR}_{10-20}$. Methods After ethical approval and informed consent, consecutive patients attending cardiac rehabilitation in a tertiary referral centre were enrolled. All patients had percutaneous coronary revascularisation. During an active stand, real-time heart rate, blood pressure and ECG recordings were taken using non-invasive digital photoplethysmography and $\mathrm{HRR}_{10-20}$ determined. Assessment of autonomic function was performed by determining speed of $\mathrm{HRR}_{10-20}$ post-orthostatic challenge. Patients with an rSs $>0$ were considered incompletely revascularised and those with an rSs of 0 fully revascularised. Demographic data were recorded and statistical analysis performed.

Results Patients $(n=53)$ comprised those with complete revascularisation $(C R)(n=37)$ and ICR $(n=16)$. In the ICR group, mean rSs was 9.4.

$\mathrm{HRR}_{10-20}$ was impaired in the ICR group $(-3 \pm 0.60)$ compared with the CR cohort $(-6.56 \pm 0.52)(p<0.0001)$. Completeness of revascularisation was strongly associated with HRR $_{10-20}$ (Pearson's correlation coefficient 0.529; $\mathrm{p}<0.0001)$. Baseline demographics did not differ significantly. Use of rate-limiting medication was similar between cohorts (beta blockers, calcium channel blockers, ivabradine).

Conclusions Our data confirm significant correlation between ICR and impaired autonomic function determined by speed of heart rate recovery. Thus, determining autonomic dysfunction post-ICR may identify those at increased mortality risk.

\section{INTRODUCTION}

Autonomic function plays a central role in control of homeostatic mechanisms governing heart rate (HR). This can be assessed clinically using various indices including: HR recovery (HRR) following either exercise or orthostatic challenge, and various metrics of HR variability

\section{Key questions}

What is already known about this subject?

- Autonomic function as determined by heart rate recovery time between 10 and $20 \mathrm{~s}\left(\mathrm{HRR}_{10-20}\right)$ has been previously shown to correlate with all-cause mortality, while incomplete revascularisation has additionally been demonstrated to correlate with allcause mortality.

What does this study add?

- Through this study, a strong correlation between autonomic function as derived from $\mathrm{HRR}_{10-20}$ and completeness of revascularisation has been determined.

How might this impact on clinical practice?

- The use of $\mathrm{HRR}_{10-20}$ may allow risk stratification to take place, and direct further interventions in order to potentially improve autonomic function and even reduce risk of mortality.

(HRV). Studies have previously shown in a non-standardised manner that HRR time following exercise (varying between 1 and $5 \mathrm{~min}$ following cessation of exercise) correlates with increased rates of mortality in various patient populations, including patients without clinically evident cardiovascular disease. ${ }^{1-3}$ HRR after orthostatic challenge has also been reported as a predictor of cardiovascular disease and mortality. HR initially increases upon moving from lying to standing position, due to inhibition of vagal tone. A peak of HR is observed at approximately $10 \mathrm{~s}$, with a reduction thereafter to around 20s. Blood pressure (BP) initially falls in this same time period; blood pools in the lower limbs, reducing venous return and therefore stroke volume, precipitating the reduction of vagal tone which contributes to the increase in HR. This pattern of HR and BP change following orthostatic challenge is shown in figure 1. ${ }^{4}$ Our group has previously demonstrated that the velocity of HRR, between 10 and $20 \mathrm{~s}\left(\mathrm{HRR}_{10-20}\right)$ following orthostatic challenge, predicts allcause mortality in adults 50 years and older (mean age 64 years) in a general population. 


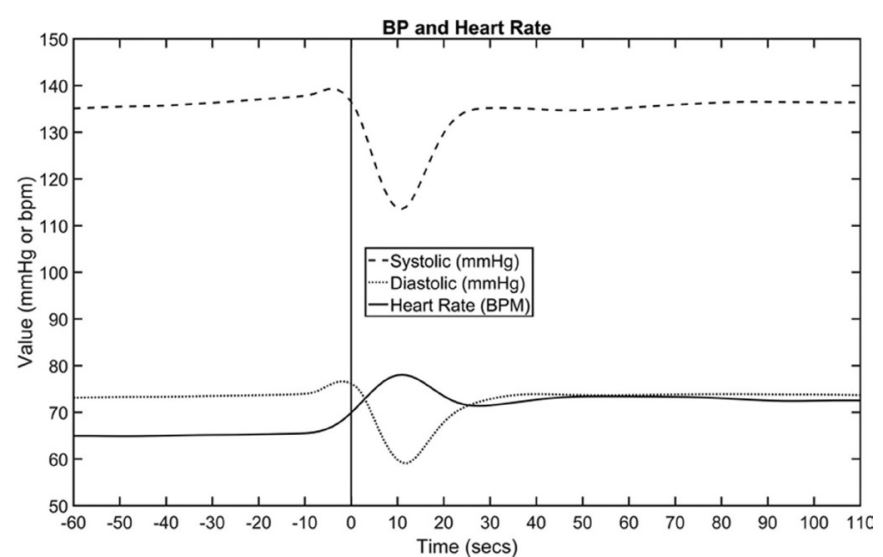

Figure 1 Mean heart rate and systolic blood pressure (SBP) and diastolic blood pressure (DBP) values across the stand (including baseline). BP, blood pressure; bpm, beats per minute.

Participants in the slowest recovery quartile were 2.3 times more likely to die within 4 years than those in the fastest recovery group, independent of other risk factors. ${ }^{4}$

Both HRV metrics and HRR have been used previously in clinical research as a surrogate marker of autonomic tone, as no direct measurements of the autonomic system are possible. This can mean that other factors can influence the extrapolated results; for example, circadian rhythm, hormones and temperature. Furthermore, time and frequency HRV indices such as PNN50 (mean number of times an hour where the change in successive normal sinus intervals exceeds $50 \mathrm{~ms}$ ), SDNN (SD of normal sinus beats), SDANN (SD of normal sinus intervals for each of the 5 min segments within a 24-hour period) and rMSSD (root mean square of successive differences between normal heartbeats), along with HRR following exercise stress testing, have demonstrated a correlation between the presence of coronary artery disease, mortality and impaired autonomic function. ${ }^{5-7}$

Incomplete cardiac revascularisation (ICR) is associated with increased 5-year mortality. Various trials have attempted to elucidate the benefit of a complete revascularisation (CR) strategy in patients with complex coronary artery disease.$^{8-11}$ The SYNTAX score is an objective, quantitative and widely used tool used to demonstrate the burden and complexity of coronary artery disease, while the residual SYNTAX score ( $\mathrm{rSs}$ ) has additionally been used to describe residual coronary artery diseaseindicative of ICR following percutaneous coronary intervention $(\mathrm{PCI}) .{ }^{12}$

Previous research has demonstrated correlation between time and frequency domain variables of HRV with percutaneous revascularisation, but the use of HRR velocity has not been previously described. ${ }^{513} 14$ Given that both impaired autonomic function and ICR are both associated with increased mortality, we hypothesised that
ICR would be associated with impaired autonomic function derived from the novel variable $\mathrm{HRR}_{10-20}$.

\section{METHODS \\ Study design}

We conducted a prospective case-control study from August 2019 until March 2020 in a tertiary referral hospital in Dublin, Ireland. The hospital is a supraregional referral centre for coronary intervention. A sample size of 48 with a ratio of $1: 2$ was calculated to detect a difference in $\mathrm{HRR}_{10-20}$ of 2 beats/min with $95 \% \mathrm{CI}$ and $90 \%$ power. Estimate of difference in $\mathrm{HRR}_{10-20}$ was based on previous studies of $\mathrm{HRR}_{10-20}{ }^{415-17}$ We aimed to recruit 60 cases (20 ICR vs $40 \mathrm{CR}$ ) over a 6 -month period. Written informed consent was obtained from all participants. Patients and the public were not involved in the design, or conduct, or reporting, or dissemination plans of the research.

\section{Study participants \\ Cases}

Cases were patients aged $>18$ years of age who had undergone PCI for stable and unstable coronary syndromes performed within the previous 3 months. Inclusion criteria included ICR determined by an rSs of $>0$, the ability to perform an active stand from supine to standing within $5 \mathrm{~s}$, the ability to provide informed consent and resting sinus rhythm. Participants were determined to be ineligible if they had a paced cardiac rhythm, atrial fibrillation, if they were unable to provide consent and if they were unable to move from supine to standing within $5 \mathrm{~s}$. Cases were recruited between 1 August 2019 and 1 March 2020.

\section{Controls}

Controls were patients aged $>18$ years of age who had undergone PCI for stable and unstable coronary syndromes performed within the previous 3 months. Inclusion criteria included CR determined by an rSs of $>0$, the ability to perform an active stand from supine to standing within $5 \mathrm{~s}$, the ability to provide informed consent and resting sinus rhythm. Participants were determined to be ineligible if they had a paced cardiac rhythm, atrial fibrillation, if they were unable to provide consent and if they were unable to move from supine to standing within 5 s. Cases were recruited between 1 August 2019 and 1 March 2020.

\section{Data collection and validation}

All eligible participants were recruited consecutively postrevascularisation through the outpatient cardiac rehabilitation programme. Participants answered a baseline semistructured questionnaire, including self-reported answers with investigator assistance to complete, containing demographical data, comorbidities including diabetes, smoking history and family history of premature coronary artery disease along with medication usage-including rate-limiting or autonomic-modulating medications (beta blockers, ivabradine, calcium channel blockers, salbutamol). Participants additionally underwent a 
physical examination including height and weight. Medical records were reviewed to determine the type of stent used, where the stent was placed and the indication for PCI.

Baseline anatomical SYNTAX score and rSs were calculated by two experienced interventional cardiologists who were blinded to the clinical outcomes and using a web-based calculator, and disagreement was resolved by consensus. Patients with an $\mathrm{rSs}>0$ were considered incompletely revascularised and those with an rSs of 0 fully revascularised.

Assessment of autonomic function was performed by determining speed of $\mathrm{HRR}_{10-20}$ post-orthostatic challenge. A pressure cuff is applied to the participant's finger, which measures phasic BP. Following a 10-minute rest period in a supine position, participants were instructed to stand promptly (within $5 \mathrm{~s}$ ). The zero time point was set at the point where the participant began to rise from supine. Beat-to-beat variability in $\mathrm{HR}$ and $\mathrm{BP}$ during the change in posture was measured via non-invasive digital photoplethysmography (Finometer, Finapres Medical systems, Arnhem, the Netherlands). Data collected were corrected for height using a height correction device applied automatically by the Finometer. During the active stand, real-time HR, BP and ECG recordings were taken and $\mathrm{HRR}_{10-20}$ extrapolated as follows: HRs after 10 and $20 \mathrm{~s}$ are calculated using a non-stationary moving average window with window width set at $\pm 5 \mathrm{~s}$ to filter the HR from the Finometer HR signal, from which the velocity of change within that time frame is calculated. Wave form graphs of ECG and phasic BP were assessed for reliability to ensure accuracy of derived physiological parameters, while additionally a signal abnormality index was used to quantify the quality of the signal data within the active stand record. This calculates the proportion of beats that are noisy within the time frame of the stand, whereby 0 implies a clean record with no detected abnormal beats and 1 implies a very noisy record with all beats identified as abnormal. All study participants were included regardless of the signal abnormality index in an intention-to-treat analysis.

\section{Active stand protocol}

Participants all underwent active stand in early afternoon between hours of 12:00 and 15:00, with measurements being taken following abstention from caffeine, nicotine and exercise for the previous 12 hours. Participants had additionally been instructed to fast for the prior 2 hours to the active stand occurring. Participants underwent active stand following approximately $10 \mathrm{~min}$ of supine rest. Baseline $\mathrm{BP}$ was calculated as the mean value between 60 and $30 \mathrm{~s}$ before stand. Onset of the stand was detected via an algorithm using data from the Finometer height correction unit. Participants were instructed to remain standing for $3 \mathrm{~min}$ following moving from supine, with continuous phasic BP and ECG tracings throughout. This protocol has been used and validated in previously published research. ${ }^{415-17}$

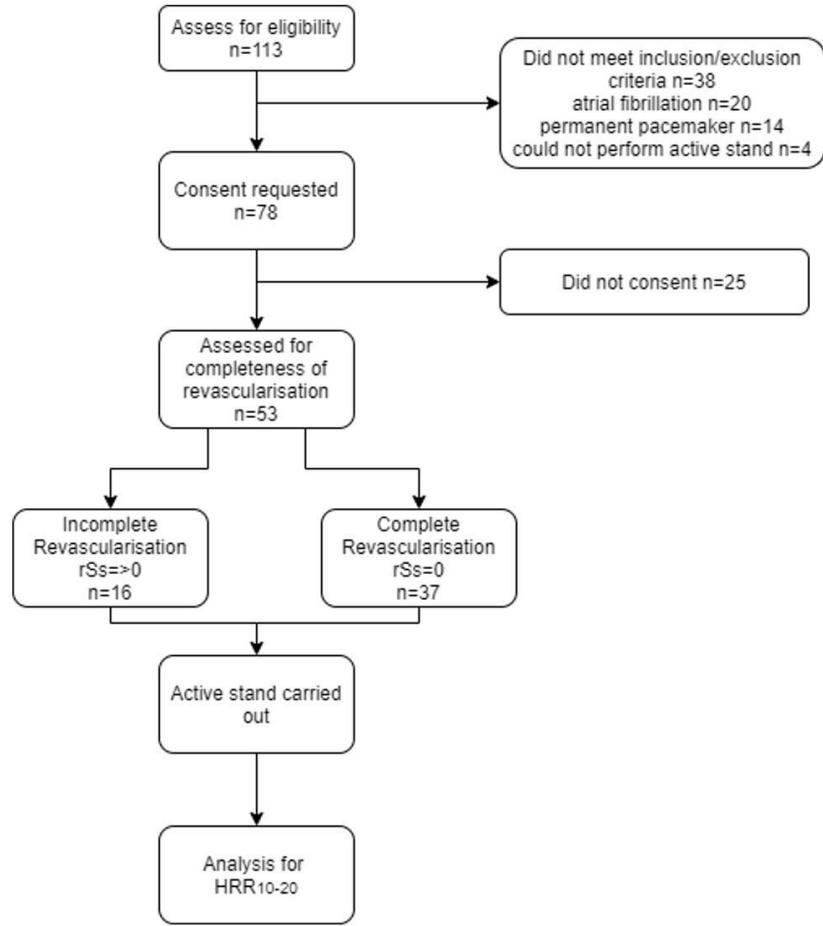

Figure 2 Flow diagram of Participant Recruitment. HRR ${ }_{10-20}$, heart rate recovery time between 10 and 20 s; rSs, residual SYNTAX score.

\section{Statistical analyses}

Statistical analysis was performed using GraphPad Prism V.9.0.2. Pearson's correlation coefficient was used to determine the relationship between $\mathrm{HRR}_{10-20}$ and ICR versus CR, Shapiro-Wilk test to assess for normality of distribution, and a Student's t-test used to determine statistical significance of the difference between the two independent groups ( $\mathrm{p} \leq 0.05$ was considered statistically significant).

\section{RESULTS}

A total of 121 participants were screened across the 6 months of recruitment, with 83 participants meeting inclusion and exclusion criteria, of whom 53 consented to participation. Of those recruited, 16 were cases with ICR and 37 were controls with CR (see figure 2).

\section{Demographic characteristics}

Cases and controls did not differ significantly in baseline demographical and background medical characteristics, including left ventricular ejection fraction (see table 1). Use of rate-limiting or autonomic-modulating medications was similar between groups.

\section{Data quality}

Signal quality index of phasic BP during active stand was similar between groups: 0.093 in the ICR group and 0.09 in the CR group. The signal quality index of ECG was also similar between the groups: 0.011 in the ICR group, 0.013 in the CR group. 
Table 1 Baseline demographics

\begin{tabular}{|c|c|c|c|}
\hline Baseline characteristics & Complete revascularisation & Incomplete revascularisation & $P$ value \\
\hline $\mathrm{n}$ & 37 & 16 & \\
\hline Age, mean (years) & 60.77 & 64.21 & 0.17 \\
\hline Age, $>65(\%)$ & 48 & 51 & 0.94 \\
\hline Male sex (\%) & 64.1 & 63.1 & 0.95 \\
\hline Height (cm) & 169.5 & 170.3 & 0.96 \\
\hline Height (kg) & 84.9 & 86.4 & 0.94 \\
\hline Never smoked (\%) & 49 & 46.4 & 0.92 \\
\hline Former smoker (\%) & 40 & 42.5 & 0.94 \\
\hline Current smoker (\%) & 11 & 11.1 & 0.97 \\
\hline Diabetic (\%) & 11 & 13.5 & 0.89 \\
\hline Hypertension (\%) & 94 & 95.3 & 0.97 \\
\hline Dyslipidaemia (\%) & 92.5 & 94 & 0.95 \\
\hline Family history of coronary artery disease (\%) & 57 & 58.1 & 0.97 \\
\hline ST-segment elevation myocardial infarction (\%) & 34.2 & 39.3 & 0.93 \\
\hline Non-ST-segment elevation acute coronary syndrome (\%) & 25.2 & 23.9 & 0.91 \\
\hline Chronic coronary syndrome (\%) & 40.6 & 36.8 & 0.86 \\
\hline Left ventricular ejection fraction (\%) & 49.7 & 50.1 & 0.91 \\
\hline Beta blocker Rx (no) & 36 & 16 & 0.93 \\
\hline Calcium channel blocker Rx (no) & 0 & 0 & \\
\hline Ivabradine Rx (no) & 3 & 1 & 0.94 \\
\hline Salbutamol Rx (no) & 1 & 0 & 0.93 \\
\hline Procedures implanting zotorolamus eluting stents (no) & 5 & 2 & 0.87 \\
\hline Procedures implanting biolimus eluting stents (no) & 2 & 1 & 0.94 \\
\hline Procedures implanting everolimus eluting stents (no) & 33 & 12 & 0.84 \\
\hline Stents implanted (no) & 1.7 & 1.8 & 0.91 \\
\hline Left main stent PCI (no) & 3 & 1 & 0.94 \\
\hline Left anterior descending $\mathrm{PCI}$ (no) & 22 & 7 & 0.90 \\
\hline Left circumflex PCI (no) & 12 & 4 & 0.89 \\
\hline Right coronary artery $\mathrm{PCl}(\mathrm{no})$ & 26 & 9 & 0.86 \\
\hline Time from revascularisation to active stand (weeks) & 10.4 & 11.1 & 0.88 \\
\hline
\end{tabular}

$\mathrm{PCl}$, percutaneous coronary intervention; $\mathrm{Rx}$, prescription.

\section{Autonomic function and revascularisation status}

HRR $_{10-20}$ was impaired in the ICR group $(-3 \pm 0.60)$ compared with the CR cohort $(-6.56 \pm 0.52) \quad(\mathrm{p}<0.0001)$ (figure 3). Completeness of revascularisation was strongly associated with $\mathrm{HRR}_{10-20}$ (Pearson's correlation coefficient $0.529 ; \mathrm{p} \leq 0.0001)$.

No significant difference was observed between groups in relation to resting or peak systolic BP, resting or peak diastolic BP and resting or peak HR, as described in table 2

\section{DISCUSSION}

Our data confirm significant correlation between revascularisation status and autonomic function determined by speed of HRR. Given the previously established correlation between impaired autonomic function and all-cause mortality and also the correlation between ICR and all-cause mortality, our data support the hypothesis that increased mortality due to ICR may be as a result of impaired autonomic function and sequelae to which this pertains.

We have also demonstrated that assessment of autonomic function may facilitate risk stratification among a high-risk group of patients who have undergone PCIs.

Our findings indicate that autonomic function correlates with completeness of revascularisation. We postulate that this may be due to myocardial ischaemia leading to homeostatic feedback through the neurocardiac system causing reduced vagal tone. In the event that myocardium is ischaemic, hypoxia and metabolic stress may cause differences in efferent signalling through the vagus nerve and cardiac plexus when compared with 


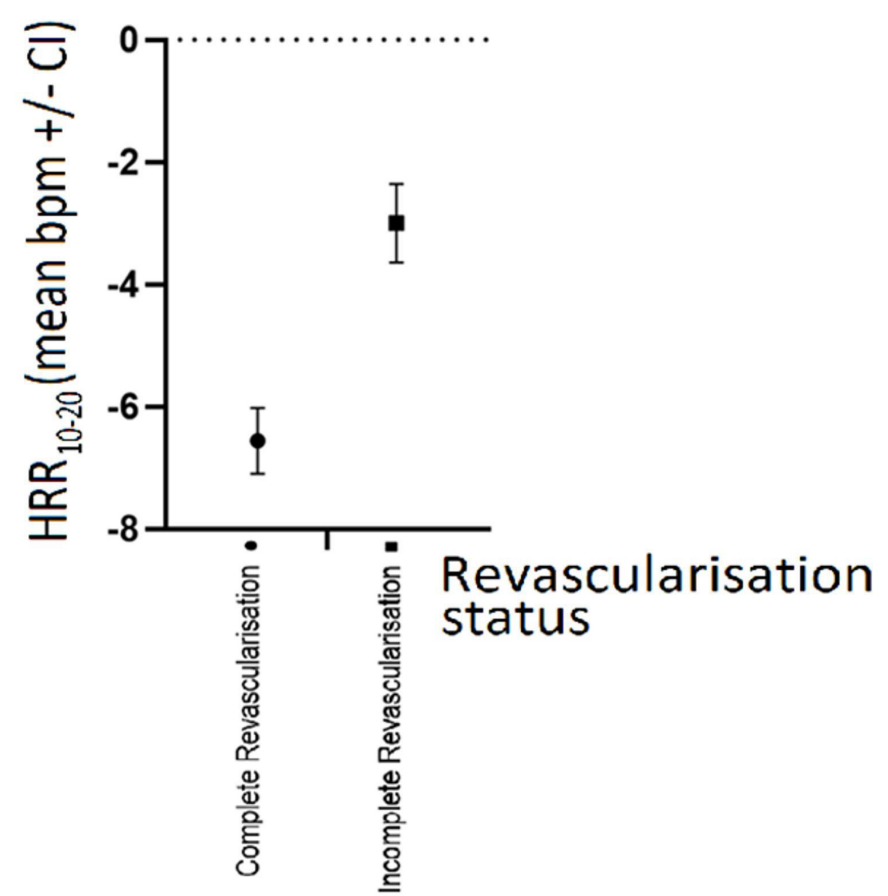

Figure 3 Impact of revascularisation status on HRR 10-20. bpm, beats per minute; $\mathrm{HRR}_{10-20}$, heart rate recovery time between 10 and $20 \mathrm{~s}$.

non-ischaemic myocardium. As a result, homeostatic afferent signalling to the sinoatrial and atrioventricular nodes may differ resulting in the observed differences in $\mathrm{HRR}_{10-20}$.

Extrapolating from these findings, we can additionally consider that overall complexity of coronary artery disease may also correlate with autonomic function. We have observed that a SYNTAX score of $>0$ correlates with impaired autonomic tone, and we suggest that given our theory that myocardial ischaemia is the causative factor in this dysregulation, we also suggest that as SYNTAX score increases, greater impairment in autonomic function may result. This theory should be tested in a larger patient cohort.

Furthermore, our postulation that ischaemia is the causative factor of impaired autonomic function leads to the theory that autonomic function may improve following coronary revascularisation, and that autonomic function could be a measure of procedural success. This additionally requires validation, and can be assessed by performing autonomic assessments prior to and post-revascularisation.

The data which we have collected correlate with results from previous research; Bonnemeier et al demonstrated that following primary PCI for ST-segment elevation myocardial infarction positively impacted on autonomic function as determined by the SDNN HRV parameter, while Sedziwy et al further showed that HRV as described by PNN50, SDNN, SDANN and rMSSD improves following PCI in patients with stable coronary artery disease. ${ }^{13}{ }^{14}$ Our data are the first to show a correlation between revascularisation status and autonomic function determined by the novel parameter $\mathrm{HRR}_{10-20}$.

The main limitation of this study is that we measured surrogate markers of autonomic function through $\mathrm{HRR}_{10}$ ${ }_{20}$. Due to other factors that can impact HR physiological indices, it may be that there is an inbuilt bias in the results being implied to autonomic function as a whole. It may be more suitable in a future study to perform multiple assessments of autonomic function beyond the HR variable which we assessed.

The case-control design of the study is also a limitation. This allowed determination that there is correlation between revascularisation status and HR autonomic variables, but does not definitively determine how they are linked. A prospective study assessing autonomic function prior to and post-revascularisation may allow determination if revascularisation itself is what improves autonomic tone. Furthermore, performing quantitative assessment of ischaemia such as perfusion imaging may validate the theory that myocardial ischaemia is the causative factor of impaired autonomic function.

Additionally, patients who had been revascularised through percutaneous intervention were primarily assessed. The impact of coronary artery bypass surgery has not been assessed; the impact of sternotomy, intensive care postoperative stay, saphenous venous grafts versus arterial grafts and other surgical variables was not reviewed as part of this study and thus our findings

Table 2 Measured physiological indices; complete revascularisation versus incomplete revascularisation cohorts

\begin{tabular}{lllc}
\hline Physiological index & Complete revascularisation & Incomplete revascularisation & P value \\
\hline HRR $_{10-20}(\mathrm{bpm})$ & $-6.56 \pm 0.52$ & $-3 \pm 0.60$ & $<0.0001$ \\
HR rest $(\mathrm{bpm})$ & $66.36 \pm 2.96$ & $66.63 \pm 5.67$ & 0.93 \\
HR peak $(\mathrm{bpm})$ & $82.74 \pm 4$ & $83.89 \pm 7.55$ & 0.77 \\
SBP rest $(\mathrm{mm} \mathrm{Hg})$ & $136.56 \pm 5.29$ & $139.53 \pm 8.38$ & 0.55 \\
SBP peak $(\mathrm{mm} \mathrm{Hg})$ & $127.31 \pm 8.97$ & $109.95 \pm 12.8$ & 0.75 \\
DBP rest $(\mathrm{mm} \mathrm{Hg})$ & $78.85 \pm 3.83$ & $80.63 \pm 5.77$ & 0.61 \\
DBP peak $(\mathrm{mm} \mathrm{Hg})$ & $69.97 \pm 5.31$ & $63.79 \pm 9.96$ & 0.25 \\
\hline
\end{tabular}

Mean values with $95 \% \mathrm{Cl}$.

bpm, beats per minute; DBP, diastolic blood pressure; HR, heart rate; HRR ${ }_{10-20}$, heart rate recovery time between 10 and 20 s; SBP, systolic blood pressure. 
cannot be extrapolated to include them. Additionally, the relative impact of percutaneous versus surgical revascularisation on autonomic function was not assessed.

Furthermore, while these data demonstrate similar left ventricular function between ICR and CR groups, we did not formally assess for left ventricular scar burden or include a significant number of participants with severely reduced left ventricular systolic function. This area may be assessed in future research with potential use of cardiac MRI to assess for burden of scar, systolic function, along with assessment of ischaemia itself to assess for correlation with $\mathrm{HRR}_{10-20}$.

Further limitations are the limited sample size along with the single-centre recruitment. Due to these limitations, it may be prudent to view the findings derived from the study as further hypothesis generating, allowing direction for future research.

Through our observations, we have raised questions which can be assessed in future research: Does autonomic function correlate with increasing complexity of coronary artery disease?; Is myocardial ischaemia the driving factor behind autonomic dysregulation?; Does revascularisation improve autonomic function?; Does percutaneous versus surgical revascularisation make a difference? These questions will require further research to answer, and may allow autonomic function to be used as a marker of procedural success in the future.

The physiology behind the homeostatic control of HR is increasingly well understood. Beyond the previously described role of autonomic variables in risk stratification, it may be possible to use physiological parameters such as $\mathrm{HRR}_{10-20}$ as surrogate markers of disease progression and measures of treatment success or regression.

\section{CONCLUSION}

The data support the hypothesis that ICR is associated with impaired autonomic function determined by $\mathrm{HRR}_{10-}$ ${ }_{20}$. This association has not previously been described and requires further study to elucidate physiological and pathophysiological cause.

Contributors RA-conception and design of the work, data collection, data analysis and interpretation, drafting article and responsible for the overall content as guarantor. PW—-data collection, data analysis and interpretation. LB - drafting article. CF-data analysis and interpretation. RAK - conception and design of the work, critical revision of the article and final approval of the version to be published. $\mathrm{AM}$ - conception and design of the work, critical revision of the article and final approval of the version to be published

Funding This work was supported by and unrestricted grant from the Royal Baggot Street Trust. CF is supported by an SFI grant.

Competing interests $\mathrm{CF}$ is working on an industry partnership grant with Endotronix.

Patient consent for publication Not required.

Ethics approval Ethical approval was confirmed by the St James's Hospital/ Tallaght University Hospital Joint Research and Ethics Committee, Dublin, Ireland (REC: 2019-07 List 27 (19)).

Provenance and peer review Not commissioned; externally peer reviewed.
Data availability statement Data are available upon reasonable request. The data that support the findings of this study are available from the corresponding author $(\mathrm{RA})$, upon reasonable request.

Open access This is an open access article distributed in accordance with the Creative Commons Attribution Non Commercial (CC BY-NC 4.0) license, which permits others to distribute, remix, adapt, build upon this work non-commercially, and license their derivative works on different terms, provided the original work is properly cited, appropriate credit is given, any changes made indicated, and the use is non-commercial. See: http://creativecommons.org/licenses/by-nc/4.0/.

\section{ORCID iDs}

Richard Armstrong http://orcid.org/0000-0002-6698-8109

Peter Wheen http://orcid.org/0000-0002-2049-471X

\section{REFERENCES}

1 Cole CR, Blackstone EH, Pashkow FJ, et al. Heart-rate recovery immediately after exercise as a predictor of mortality. $N$ Engl J Med 1999;341:1351-7.

2 Morshedi-Meibodi A, Larson MG, Levy D, et al. Heart rate recovery after treadmill exercise testing and risk of cardiovascular disease events (the Framingham heart study). Am J Cardiol 2002;90:848-52.

3 Lipinski MJ, Vetrovec GW, Froelicher VF. Importance of the first two minutes of heart rate recovery after exercise treadmill testing in predicting mortality and the presence of coronary artery disease in men. Am J Cardiol 2004;93:445-9.

4 McCrory C, Berkman LF, Nolan $\mathrm{H}$, et al. Speed of heart rate recovery in response to orthostatic challenge. Circ Res 2016;119:666-75.

5 La Rovere MT, Bigger JT, Marcus FI, et al. Baroreflex sensitivity and heart-rate variability in prediction of total cardiac mortality after myocardial infarction. ATRAMI (autonomic tone and reflexes after myocardial infarction) Investigators. Lancet 1998;351:478-84.

6 Mølgaard H, Sørensen KE, Bjerregaard P. Attenuated 24-h heart rate variability in apparently healthy subjects, subsequently suffering sudden cardiac death. Clin Auton Res 1991;1:233-7.

7 Camm AJ, Pratt CM, Schwartz PJ, et al. Mortality in patients after a recent myocardial infarction: a randomized, placebo-controlled trial of azimilide using heart rate variability for risk stratification. Circulation 2004;109:990-6.

8 Vieira Ricardo D'Oliveira, Hueb W, Gersh BJ, et al. Effect of complete revascularization on 10-year survival of patients with stable multivessel coronary artery disease: MASS II trial. Circulation 2012;126:S158-63.

9 Wald DS, Morris JK, Wald NJ, et al. Randomized trial of preventive angioplasty in myocardial infarction. $N$ Engl $\mathrm{J}$ Med 2013;369:1115-23.

10 Gershlick AH, Khan JN, Kelly DJ, et al. Randomized trial of complete versus lesion-only revascularization in patients undergoing primary percutaneous coronary intervention for STEMI and multivessel disease: the CvLPRIT trial. J Am Coll Cardiol 2015;65:963-72.

11 Engstrøm T, Kelbæk H, Helqvist S, et al. Complete revascularisation versus treatment of the culprit lesion only in patients with STsegment elevation myocardial infarction and multivessel disease (DANAMI-3-PRIMULTI): an open-label, randomised controlled trial. Lancet 2015;386:665-71.

12 Farooq V, Serruys PW, Bourantas CV, et al. Quantification of incomplete revascularization and its association with five-year mortality in the synergy between percutaneous coronary intervention with Taxus and cardiac surgery (SYNTAX) trial validation of the residual SYNTAX score. Circulation 2013;128:141-51.

13 Bonnemeier $\mathrm{H}$, Hartmann F, Wiegand UK, et al. Heart rate variability in patients with acute myocardial infarction undergoing primary coronary angioplasty. Am J Cardiol 2000;85:815-20.

14 Sedziwy E, Olszowska M, Tracz W, et al. [Heart rate variability in patients treated with percutaneous transluminal coronary angioplasty]. Przegl Lek 2002;59:695-8.

15 Finucane C, O'Connell MDL, Fan CW, et al. Age-Related normative changes in phasic orthostatic blood pressure in a large population study: findings from the Irish longitudinal study on ageing (TILDA). Circulation 2014;130:1780-9.

16 McCrory C, Berkman LF, Moore PV, et al. What explains socioeconomic differences in the speed of heart rate recovery to postural challenge? J Gerontol A Biol Sci Med Sci 2017;72:1717-23.

17 Finucane C, van Wijnen VK, Fan CW, et al. A practical guide to active stand testing and analysis using continuous beat-to-beat noninvasive blood pressure monitoring. Clin Auton Res 2019;29:427-41. 